\title{
FORÇAS DE CORTE ORTOGONAL 90-90 EM TRÊS ESPÉCIES DE MADEIRA DE EUCALIPTO DO ESTADO DE SÃO PAULO
}

\author{
Antônio Carlos Néri ${ }^{1}$, Raquel Gonçalves ${ }^{2}$ \& Roger E. Hernandez ${ }^{3}$
}

\begin{abstract}
RESUMO
No projeto de ferramentas de corte e potência de máquinas que compõem uma serraria, a força de corte ortogonal é o principal parâmetro utilizado. Estas forças variam em função da espécie, das condições da madeira a ser usinada (densidade, teor de umidade e orientação das fibras), da geometria da ferramenta (afiação, ângulo de cunha, espessura e largura da lâmina) e das condições de usinagem (ângulo de ataque, velocidade de avanço, velocidade de corte, espessura e largura de corte). O corte 90-90 é de extrema importância, pois é muito utilizado no processamento primário da madeira. As espécies de reflorestamento, em especial as de eucalipto, vêm assumindo, dia-a-dia, significativa importância no mercado nacional e de exportação. Este trabalho apresenta resultados de ensaios de corte ortogonal 90-90, realizado utilizando-se corpos de prova de Eucalyptus das espécies citriodora, saligna e grandis. Os resultados mostram a influência dos parâmetros de usinagem adotados nas forças de corte e indicam a importância do seu conhecimento para a otimização dos processos de desdobro da madeira.
\end{abstract}

Palavras-chave: Eucalyptus, usinagem, força de corte

\section{ORTHOGONAL 90-90 CUTTING FORCES FOR THREE WOOD SPECIES OF EUCALYPTUS}

\begin{abstract}
Orthogonal cutting force is a fundamental parameter to design cutting tools and to determine to the required power necessary to operate a sawmill. This force varies within different species, and as a function of the wood condition (density, moisture content, fiber orientation), tool shape (sharpness angle, clearance angle, blade thickness and width) as well as of the cutting equipment (rake angle, forward velocity, cutting speed, chip thickness and width). The 90-90 cutting is extremely important because it occurs during end-grain cutting. The reforestation species, in particular the eucalyptus, are acquiring increasing importance in the Brazilian domestic and export timber market. This paper reports the results of 90-90 orthogonal cutting tests, conducted using specimens from three species of Eucalyptus: citriodora, saligna and grandis. The results show the influence of the machining parameters in the cutting forces and indicate its relevance in order to optimize the wood machining process.
\end{abstract}

Key words: Eucalyptus, machining, cutting force

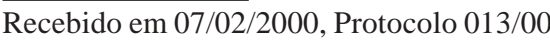

${ }^{1}$ Estudante de pós-graduação, UNICAMP,Cidade Universitária Zeferino Vaz s/n, Campinas, SP. Fone: (0xx35) 821 3751. E-mail: acneri@agr.unicamp.br

${ }^{2}$ Prof. Dra., Faculdade de Engenharia Agrícola, UNICAMP. CEP 13083 - 970, Campinas, SP. Fone: (0xx 19) 788 1044. E-mail: raquel@agr.unicamp.br

${ }^{3}$ Prof. Ph.D., Faculdade de Ciências Florestais e da Madeira. Pavillon Abitibi Price G1K - 7P4. Université Laval, Q.C Canadá. Fone: (418)656 5852.E-mail: roger.hernandez@sbf.ulaval.ca 


\section{INTRODUÇÃO}

O emprego de espécies de reflorestamento, como o eucalipto e o pinus, vem crescendo no setor madeireiro no Brasil, em função da necessidade de substituição da madeira de espécies provenientes de florestas nativas tradicionalmente utilizadas pelo setor da construção civil e moveleiro.

Apesar de grandes avanços tecnológicos em várias áreas, o setor madeireiro necessita, ainda, de conhecimentos básicos na área de usinagem. Em muitas serrarias e indústrias madeireiras, o desdobro e o processamento da madeira ainda são realizados de maneira empírica e o dimensionamento do equipamento de corte, quando não compatível com a espécie processada, afeta diretamente os resultados dos processos de usinagem, elevando significamente o custo, o consumo de energia para o processamento, o tempo de processamento e a qualidade final do produto processado.

A madeira, por ser um material heterogêneo e anisotrópico (apresenta propriedades físico/mecânicas diferentes nos planos radial, tangencial e longitudinal) possui muitos fatores a serem considerados na usinagem. Em geral, as espécies de eucalipto requerem técnicas de usinagem mais específicas, por apresentarem características inerentes à espécie, como tensões de crescimento, fibras retorcidas, alta densidade, dureza etc. Somente a partir do conhecimento de conceitos fundamentais do comportamento dessas espécies durante o corte, será possível melhor compreensão dos processos de usinagem, para posterior repasse de tecnologia ao setor produtivo. Tais conhecimentos reduziriam, também, os problemas ainda existentes quanto à aceitação dessas espécies no mercado nacional.

Para melhor compreensão do comportamento da madeira nos diferentes processos de corte, um dos parâmetros fundamentais a se estudar são as forças implicadas durante a usinagem. Um requisito básico para se determinar se uma espécie de madeira é apta para determinado uso, é o conhecimento e análise de seu comportamento durante a usinagem, cujos processos incluem serramento, desengrosso, torneamento, lixamento e furação; algumas dessas operações podem ser encontradas no processamento primário e outras, principalmente no processamento secundário da madeira. Todos os tipos de corte envolvem um processo de tensão de ruptura, enquanto a força é transmitida à madeira por meio de ferramenta de corte, seja por ação manual ou mecânica. A orientação e a direção das forças são controladas pelo tipo de ferramenta, pelo direcionamento dado pelo carpinteiro ou pela máquina de corte, em que a forma da ferramenta e sua direção de movimento determinam a maneira como os esforços se produzem e como estes são suportados pela madeira e, portanto, influem diretamente no tipo de ruptura.

As forças de corte requeridas durante o processo de usinagem têm grande importância no projeto da geometria da ferramenta de corte e no dimensionamento das máquinas que compõem uma serraria. Essas forças de corte, por sua vez, variam com a espécie de madeira, com a direção das fibras, com a direção de corte, com a afiação da ferramenta de corte e com outras variáveis relacionadas à madeira (densidade, umidade etc.), à ferramenta de corte (ângulo e geometria da ferramenta) e às condições de corte (ângulo de ataque, velocidade de corte, espessura e largura de corte etc). Uma análise da literatura mostra que não há muita informação disponível a respeito das forças requeridas no processamento das diferentes espécies de eucalipto no Brasil. O objetivo desta pesquisa foi avaliar as forças de corte ortogonal 90-90 das espécies de Eucalyptus grandis, Eucalyptus saligna e Eucalyptus citriodora. A influência dos parâmetros de corte, ângulo de ataque, espessura de corte, densidade, direção de corte e orientação (radial e tangencial) foi avaliada.

\section{Corte}

O corte é definido como a ação do fio da aresta cortante de uma ferramenta em uma peça produzindo cavacos de dimensões e espessuras variáveis. Dependendo do processo de corte executado, tem-se o corte periférico ou o corte ortogonal.

O corte periférico é produzido por cortes sucessivos, feito por arestas cortantes instaladas na periferia de um cabeçote porta-ferramentas. As arestas cortantes das ferramentas são dispostas de maneira a se obter um mesmo cilindro de corte. Exemplo de corte periférico é o executado pela plaina desengrossadeira.

O corte ortogonal é um caso particular de corte periférico em que o diâmetro do suporte das facas tende ao infinito. Exemplo de corte ortogonal é o executado pela serra de fita. Neste trabalho, o foco de interesse foi o corte ortogonal 90-90.

O corte ortogonal é definido como sendo a situação em que o fio da ferramenta de corte é perpendicular à direção de movimento relativo entre a ferramenta e a peça de madeira e quando a superfície obtida é paralela àquela antes do corte. Máquinas tais como a serra de fita, serra circular, tornos e plainas, podem ser estudadas utilizando-se os princípios do corte ortogonal. Mackenzie (1960) propôs uma notação com dois numerais para definir as diferentes situações que se podem apresentar durante o corte ortogonal da madeira, em que o primeiro número representa o ângulo entre o fio de corte da ferramenta e a fibra da madeira, e o segundo indica o ângulo entre a direção de corte e a fibra da madeira. Esta notação define as três principais situações, ou seja, cortes 90-0, 90-90 e 0-90 (Figura 1); o corte 90-0 é encontrado em processos de corte, como os realizados pelas plainas desengrossadeiras e, também, em cortes de lâminas na direção paralela às fibras. $\mathrm{O}$ trabalho da serra de fita é um caso típico de corte 90-90. As forças de corte variam em função do tipo de corte e, normalmente, as forças obtidas para o corte 90-90 são mais elevadas que para o corte 90-0. O tipo de corte 0-90 é o que requer menores energias para o corte (Koch, 1985). Diferentes componentes de forças podem ser medidas durante o corte e ser vistas na Figura 1.

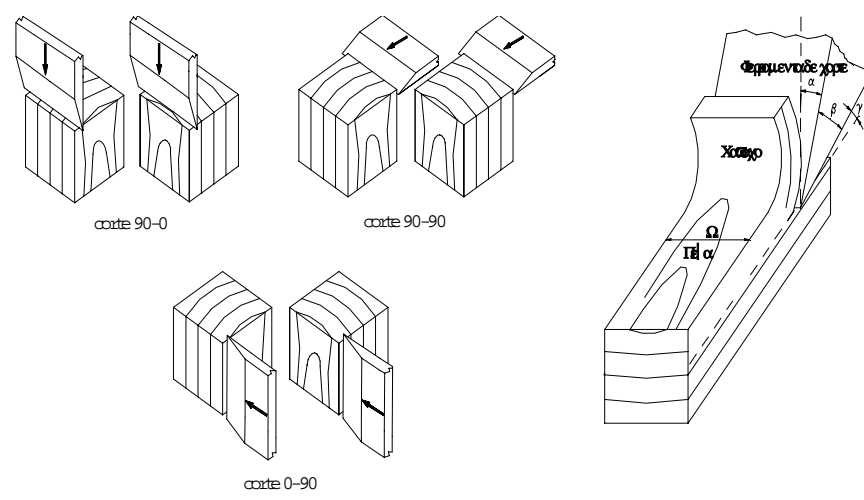

Figura 1. Principais tipos de corte ortogonal, ângulos de corte e componentes das forças (Néri et al, 1999) 


\section{Métodos de medida das forças de corte}

Existem vários métodos para se determinar as componentes das forças de corte durante o processamento da madeira. Gonçalves (1993) apresentaram um método para medir estas forças em duas direções ortogonais, ou seja, as componentes paralela $F_{p}$ e normal $F_{n}$ (Figura 1). Woodson (1979) utilizou um dinamômetro similar para avaliar o corte de 22 espécies de folhosas de zona temperada. Alguns métodos permitem a medida simultânea das três componentes principais das forças de corte, incluindo-se, neste caso, a componente lateral, salientando-se que o dinamômetro construído por King \& Foschi (1969) é um exemplo deste caso e consiste de dois anéis ortogonais providos de um sistema de 12 extensômetros, fixados de maneira tal que, teoricamente, não existe nenhuma sensibilidade cruzada ou dependência sobre o ponto de aplicação da carga. Este tipo de dinamômetro foi o adotado para realização da parte experimental deste trabalho.

\section{Corte ortogonal 90-90}

O corte 90-90 é de grande interesse prático pois se trata do tipo de corte realizado pela serra de fita de corte longitudinal, corte este amplamente utilizado nas serrarias e onde o fio da ferramenta deve separar o cavaco longitudinalmente, ou seja, a ferramenta deve separar a estrutura celular na direção perpendicular à fibra. A formação do cavaco é precedida de deformação por cisalhamento e a ruptura ocorre devido à flexão; posteriormente, este cavaco desliza e se move para fora da face de corte, formando uma espécie de cordão composto de pequenos segmentos retangulares (Hoadley, 1980). Tendo em vista que a ferramenta de corte deve separar as fibras perpendicularmente, um pequeno ângulo de ataque deforma, de maneira acentuada, a madeira, em compressão perpendicular às fibras durante o corte; efeito similar é obtido quando a ferramenta não está bem afiada, cujas condições são favoráveis à obtenção de defeitos produzidos pelo corte incompleto das fibras, caso em que as fibras são flexionadas na superfície, causando fendilhamento abaixo do plano de corte. A utilização de grandes ângulos de ataque e de ferramentas bem afiadas minimiza os danos superficiais causados pelo corte na peça (Mackenzie, 1960; Hoadley, 1980).

$\mathrm{O}$ dente da serra de fita tem menor espessura que a peça a ser cortada; sendo assim, deve-se separar e cortar as faces laterais para que passe livremente dentro da ranhura de corte. Para evitar o atrito da serra de fita com as superfícies laterais de corte, os dentes devem ser mais largos na ponta, cujo procedimento é denominado "trava" do dente; sua espessura é maior que a da fita.

Nos casos das serras circulares, a condição de corte se aproxima do tipo 90-90, quando o disco corta o mais próximo possível de sua parte central, pois quando a serra está ajustada para fazer um sulco (ranhura) raso, os dentes da mesma trabalham em uma situação de corte próximo à do tipo 90-0 (Hoadley, 1980).

\section{MATERIAL E MÉTODOS}

\section{Material}

- Madeira de eucalipto grandis, saligna e citriodora. Estas espécies foram selecionadas com o objetivo de abranger um intervalo de densidade, estando classificadas dentro de três classes de resistências da norma NBR 7190/97 (ABNT, 1997).

- Dinamômetro de anéis ortogonais, proposto por King \& Foschi (1969) com capacidade de carga de $2000 \mathrm{~N}$ para as direções X e Z e 3336N para a direção Y.

- Fresadora provida de mesa com movimento vertical e horizontal.

- Carta de aquisição de dados Strawberry mini-16, conectada a um computador PC.

- Dentes adaptados aos diferentes ângulos estudados.

\section{Métodos}

Dezesseis (16) peças de 150 x 150 x $1000 \mathrm{~mm}$ foram cortadas, procedentes de três árvores das espécies estudadas, obtidas do horto-florestal de Itirapina, Rio Claro, SP, e cortadas no Laboratório de Madeiras e Estruturas de Madeiras da Escola de Engenharia de São Carlos (LAMEM). As peças foram tratadas com produto anti-fúngico, embaladas com plástico e enviadas à Universidade Laval, Canadá. Corpos de prova foram preparados para a medida das forças de corte na direção 90-0, 90-90 e da densidade, de acordo com o esquema mostrado na Figura 2. A preparação dos corpos de prova seguiu dois tipos de orientação: tangencial e radial. As dimensões dos corpos de prova foram de $6 \mathrm{~mm}$ (direção radial ou tangencial) por $75 \mathrm{~mm}$ (direção radial ou tangencial) por $75 \mathrm{~mm}$ (longitudinal) e a madeira foi conservada no estado verde durante os ensaios de forças de corte e densidade.

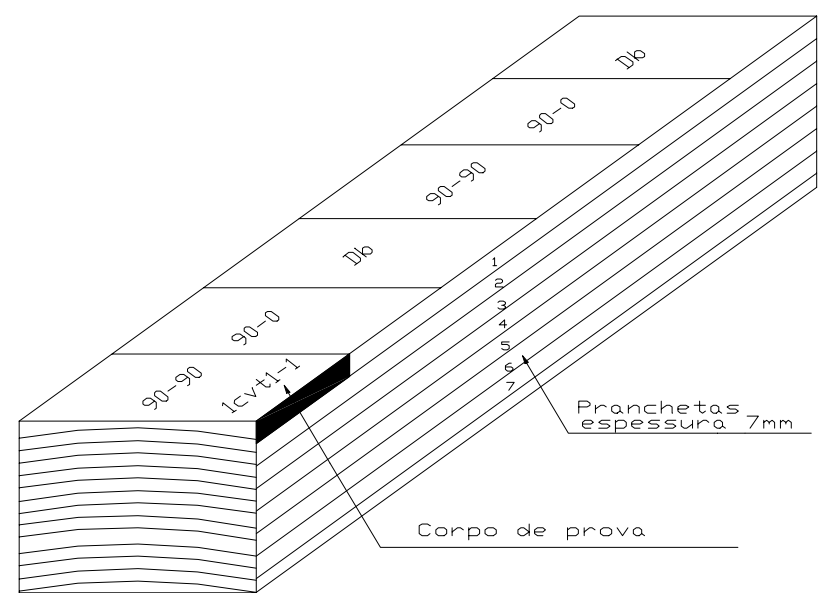

Figura 2. Exemplo de preparação de corpos de prova de orientação tangencial

\section{Ensaios para medida das forças de corte}

Antes da realização dos ensaios principais foram necessárias diversas etapas de trabalho para a definição de importantes parâmetros e para uma avaliação inicial do comportamento geral do ensaio.

Uma seqüência de ensaios preliminares permitiu identificar-se a magnitude da força de corte e mostrou a necessidade de se tratar, de maneira diferenciada, o corte ortogonal 90-0 e o 90-90.

No corte ortogonal 90-90, os ensaios preliminares mostraram uma grande dificuldade de obtenção de boa qualidade de cavaco e de superfície com a utilização de ferramenta de corte tipo faca. A qualidade de superfícies afetava os resultados das forças e, por esta razão, optou-se pelo uso de uma ferramenta de corte tipo dente (Figura 3). 


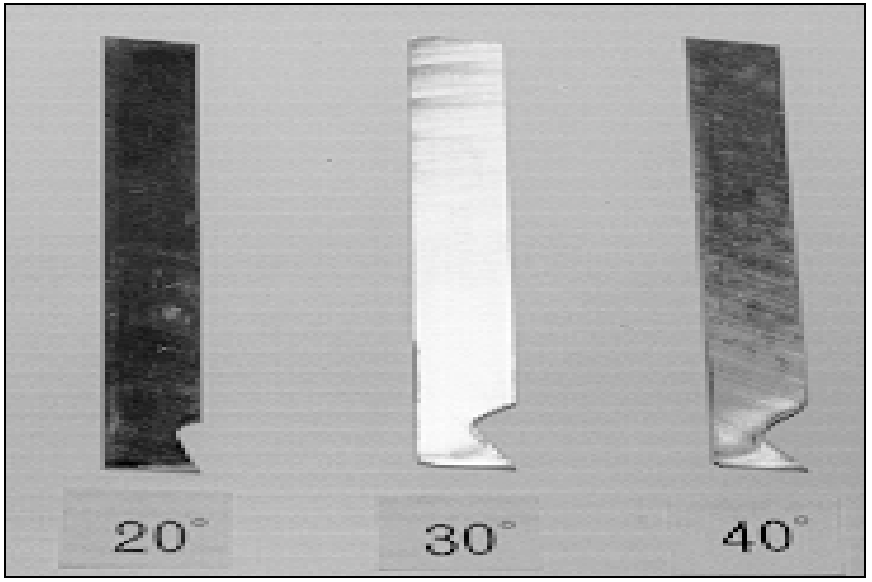

Figura 3. Ferramenta de corte tipo dente, utilizada no corte 90-90

Os ensaios preliminares evidenciaram, ainda, a necessidade da correção da superfície de corte entre uma passada e outra. Tendo em vista as espessuras de corte adotadas, a superfície de corte afetada por microfissuras a qual afetava o valor real da força medida, adotou-se a correção da superfície com uma serra radial tipo destopadeira.

Os ensaios foram realizados empregando-se quatro espessuras de corte: $0,38,0,76,1,14$ e 1,52 mm e três ângulos de ataque: $20^{\circ}, 30^{\circ} \mathrm{e} 40^{\circ} \mathrm{e}$ a velocidade de corte foi de $300 \mathrm{~mm} \mathrm{~min}^{-1}$. Duas passadas consecutivas foram realizadas para cada espessura de corte, com a finalidade de se estimar melhor o valor médio, enquanto as forças eram registradas simultaneamente durante o ensaio, nas três direções principais: paralela $\left(\mathrm{F}_{\mathrm{p}}\right)$; normal $\left(\mathrm{F}_{\mathrm{n}}\right)$ e lateral $\left(\mathrm{F}_{1}\right)$, utilizando-se a carta de aquisição de dados, que registrava 25 leituras por segundo. Um exemplo de registro é apresentado na Figura 4. Com os dados obtidos, o valor de força de corte máxima, média e mínima, foi calculado para cada ensaio.

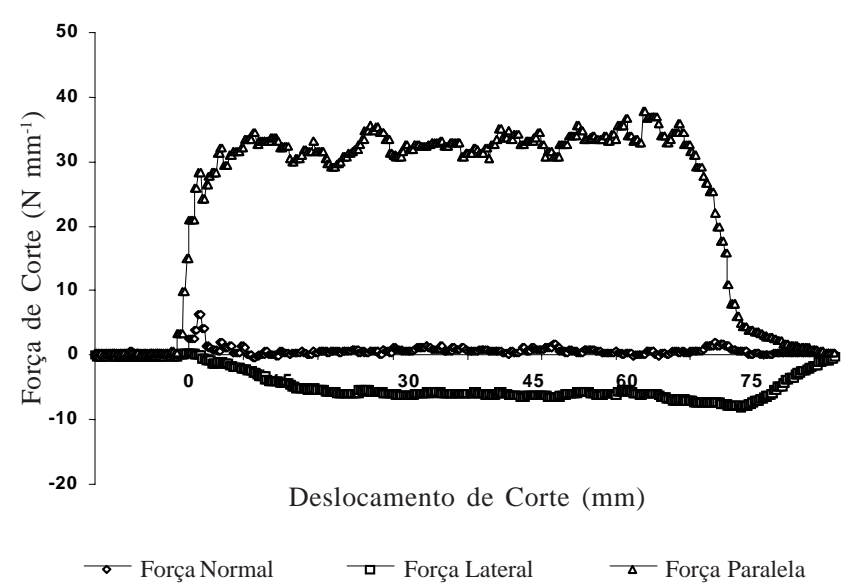

Figura 4. Exemplo de registro do gráfico com a carta de aquisição de dados

\section{Determinação da densidade e umidade da madeira}

Corpos de prova pareados àqueles utilizados para o ensaio de corte, serviram para determinar a densidade e o teor de umidade da madeira. $\mathrm{O}$ volume no estado saturado do corpo de prova foi determinado pelo método de imersão em água desionizada, tomando-se o peso em balança de $0,01 \mathrm{~g}$ de precisão.
A massa seca foi obtida após permanência dos corpos de prova em estufa a $103 \pm 2^{\circ} \mathrm{C}$, por um período de $24 \mathrm{~h}$, e a densidade básica foi então calculada pela relação massa seca/volume saturado.

\section{RESULTADOS E DISCUSSÃO}

O teor de umidade da madeira variou de 81 a $120 \%$ no eucalipto grandis, de 63 a 99\% no eucalipto saligna e de 35 a $47 \%$ no eucalipto citriodora. As forças de corte correspondem, então, ao estado verde. A densidade básica foi de $0,415,0,564$ e $0,912 \mathrm{~g} \mathrm{~cm}^{-3}$ respectivamente para o eucalipto grandis, saligna e citriodora. Esta grande amplitude de valores de densidade permitirá estudar-se a possibilidade de serem estabelecidas equações para predizer as forças de corte em função da densidade da madeira; estas relações, se confiáveis, serão de muita utilidade para extrapolar os resultados para outras espécies de eucalipto. Os valores de força de corte foram sumarizados em quadros, em que a Tabela 1 apresenta o resumo dos valores médios em $\mathrm{N} \mathrm{mm}^{-1}$ da força de corte normal, lateral e paralela para cada condição de corte; estas condições, por sua vez, incluem três espécies de eucalipto, três ângulos de ataque, quatro espessuras de corte e duas orientações do corpo de prova e cada uma das condições de corte foi realizada com 20 repetições (corpos de prova).

Segundo a literatura, no corte ortogonal 90-90 cavacos uniformes são obtidos quando o ângulo de ataque varia de 30 a $40^{\circ}$ e quando o instrumento de corte tem boa qualidade de afiação (Koch 1995). Se isto é verdade, as relações Fp(máxima)/Fp(média) devem manter-se constantes dentro de uma mesma espécie, para todas as espessuras de corte e ângulos de ataque. Os resultados obtidos neste trabalho confirmam tal expectativa. Existe uma pequena variação desta relação entre as espécies, mas dentro da espécie a relação não varia significativamente nem com a espessura de corte nem com o ângulo de ataque. Para o corte na direção tangencial, as forças máximas encontradas foram $13 \%$ maior que a força média para a espécie grandis, $17 \%$ maior que a força média para a espécie saligna e $20 \%$ maior que a força média para a espécie citriodora.

Para o corte radial, estas relações continuaram sendo constantes dentro da espécie, mas cresceram para $26 \%$ maior que a força média para o eucalipto grandis, $23 \%$ maior que a força média para o eucalipto saligna e $24 \%$ para o eucalipto citriodora. Woodson (1979) obteve, em média, um valor de força média $23 \%$ maior que a força média para as 22 espécies de dicotiledôneas pesquisadas.

Tendo em vista que a relação foi invariável e pequena, espera-se que o gráfico do ensaio seja razoavelmente constante (sem muitos picos) e que os gráficos que representam as relações entre forças e espessura de corte tenham tendência linear para todos os ângulos de ataque. Os gráficos apresentados nas Figuras 4 e 5 confirmam este resultado.

\section{Relação das forças com as espessuras de corte e densidade}

Força paralela: A força paralela média aumenta com o aumento da espessura de corte, com o aumento da densidade básica e com a diminuição do ângulo de ataque para todas as espécies. No caso dos corpos de prova tangenciais, a relação entre força paralela e espessura de corte e força paralela e densidade, foi 
Tabela 1. Valores da força de corte paralela $-\mathrm{F}_{\mathrm{p}}\left(\mathrm{N} \mathrm{mm}^{-1}\right)^{*}$ obtidos em corte ortogonal 90-90 na direção radial, em função da espessura de corte (e) e ângulo de ataque ( $\alpha$ ) para madeira de eucalipto das espécies grandis, saligna e citriodora, na condição saturada

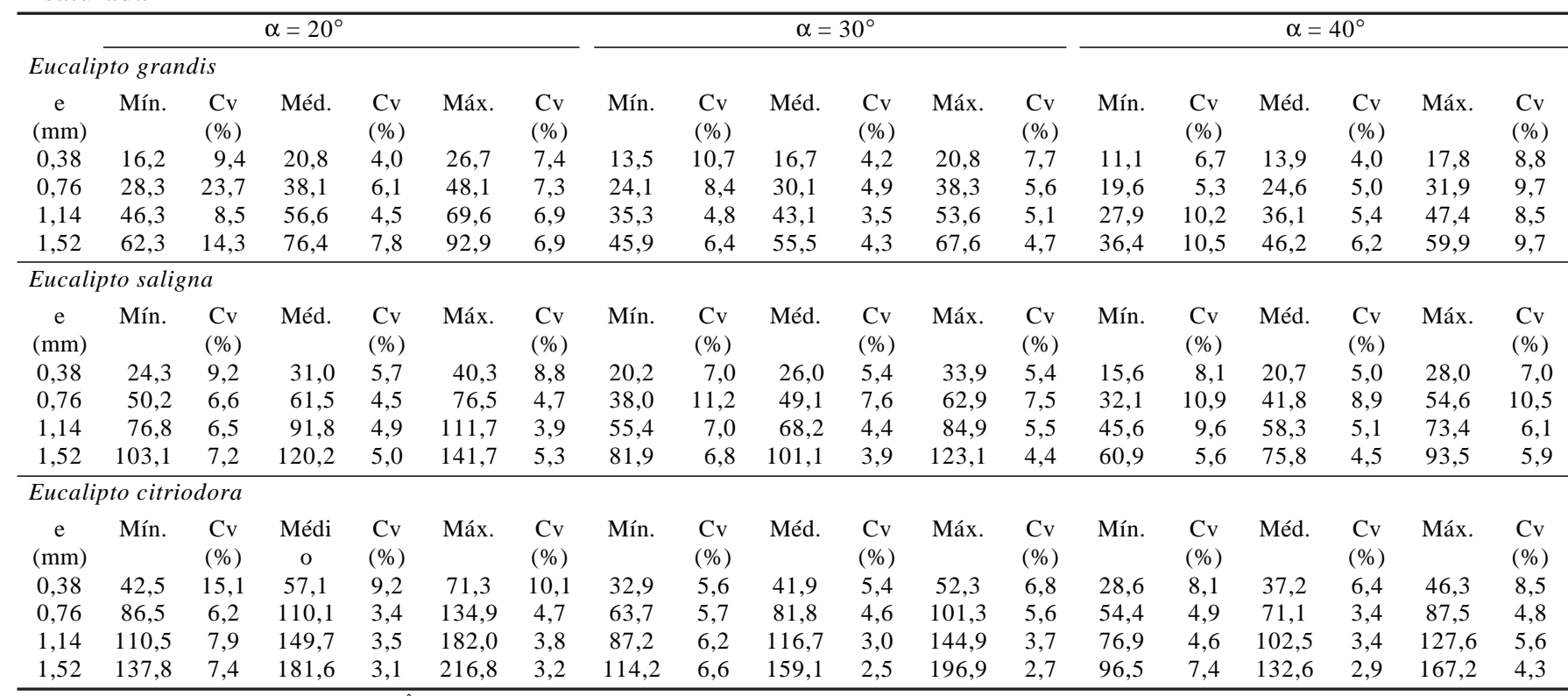

* Cada valor corresponde à média de 20 repetições. Ângulos do dente para os respectivos ângulos de ataque $\beta=63^{\circ}\left(\alpha=20^{\circ}\right) ; \beta=53^{\circ}\left(\alpha=30^{\circ}\right) ; \beta=43^{\circ}\left(\alpha=40^{\circ}\right)$; ângulo livre - $\gamma=7^{\circ}$ para todos os casos. Velocidade de corte $=30 \mathrm{~mm} \mathrm{~min}^{-1}$
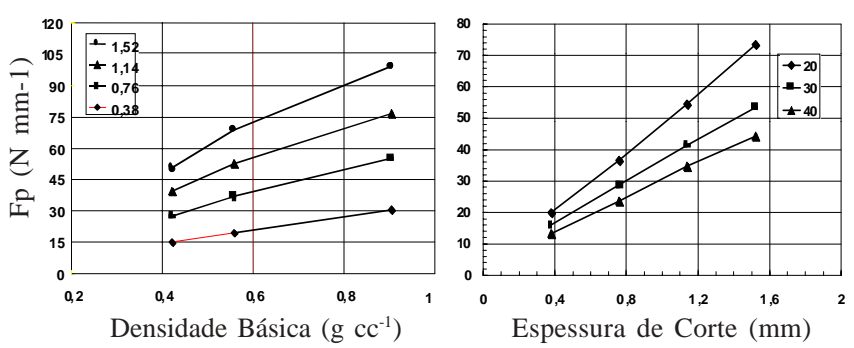

Figura 5. $\mathrm{F}_{\mathrm{N}}$ média no corte 90 - 90 em função da densidade e espessura no corte tangencial $\alpha=300$ e $\mathrm{F}_{\mathrm{P}}$ média no corte 90-90, em função da espessura, e ângulo de ataque no corte radial com eucalipto grandis

linear; já para os corpos de prova radiais, esta relação apresentou tendência linear, mas houve desvios para as maiores espessuras e menores ângulos de ataque, caso em que é possível que a correção da superfície realizada para eliminar a zona afetada pelo primeiro corte, tenha sido insuficiente. Este resultado ocorreu principalmente nas situações onde se tem as de maiores forças de corte, ou seja, com ângulos de ataque de $10^{\circ}$ e $20^{\circ}$ e grandes espessuras de corte (Figuras 6).

Força normal: De acordo com Woodson (1979) a força normal média para o corte $90-90$ é positiva para madeiras com densidades básicas menores que $0,50 \mathrm{~g} \mathrm{~cm}^{-3}$ e negativas para madeiras com densidades básicas maiores que $0,50 \mathrm{~g} \mathrm{~cm}^{-3}$. Os resultados obtidos neste trabalho mostram que, para a espécie grandis, cuja densidade básica média foi de $0,415 \mathrm{~g} \mathrm{~cm}^{-3}$, a força normal média foi sempre positiva somente para o menor ângulo de ataque $\left(20^{\circ}\right)$ e negativa para os demais ângulos estudados. Para a espécie citriodora, cuja densidade média foi de $0,912 \mathrm{~g} \mathrm{~cm}^{-3}$, as forças normais médias foram sempre negativas e, para a espécie saligna, cuja densidade média foi de $0,564 \mathrm{~g} \mathrm{~cm}^{-3}$, houve um único valor médio positivo, obtido no ângulo de ataque de $10^{\circ}$, tanto na direção radial como na tangencial.

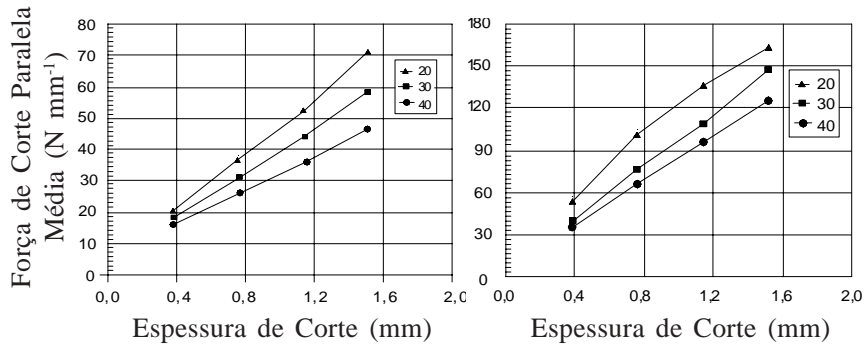

Figura 6. $\mathrm{F}_{\mathrm{N}}$ média no corte 90-90, em função da espessura, e ângulo de ataque no corte tangencial com eucalipto grandis, e $\mathrm{F}_{\mathrm{p}}$ média no corte 90-90, em função da espessura, e ângulo de ataque no corte radial com eucalipto citriodora

Não se pode afirmar que o resultado obtido neste trabalho seja discrepante dos resultados obtidos por Woodson (1979) pois as espécies grandis e saligna tinham densidades muito próximas aos limites adotados na conclusão do autor. Este resultado mostra que, provavelmente, não se possa definir o limite de $0,50 \mathrm{~g} \mathrm{~cm}^{-3}$ com tanta exatidão.

A influência da densidade na força normal aumentou com o aumento do ângulo de ataque e da espessura de corte (Figura 7).

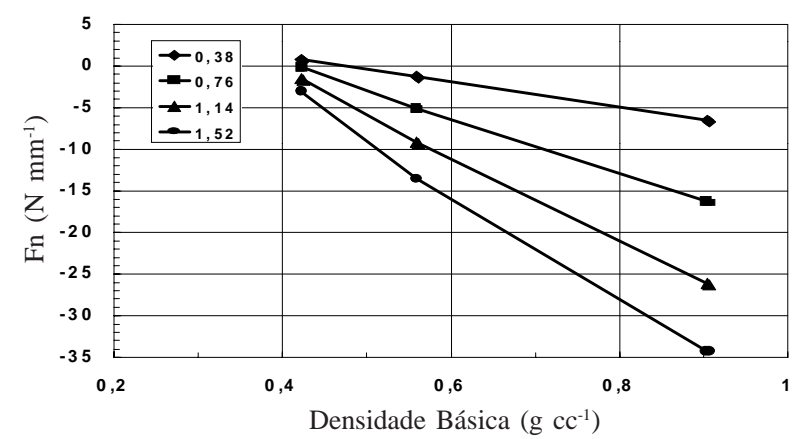

Figura 7. $\mathrm{F}_{\mathrm{N}}$ média no corte 90-90, em função da desnsidade, e ângulo da espessura no corte tangencial $\alpha=300$ 


\section{CONCLUSÕES}

1. Em geral, a força de corte paralela aumenta com o aumento da densidade. Este efeito é dependente do ângulo de ataque utilizado.

2. Os cavacos obtidos são uniformes para todas as espessuras e todos os ângulos de ataque. A força paralela máxima foi, em média, $24 \%$ maior que a força paralela média para os corpos de prova radiais e $17 \%$ maior que a força paralela média para os corpos de prova tangenciais.

3. A força paralela aumenta com o aumento da espessura de corte. Este efeito cresce com o decréscimo do ângulo de ataque.

4. A força de corte paralela decresce com o aumento do ângulo de ataque.

5. A força de corte paralela aumenta com o aumento da densidade. Este efeito é mais acentuado para ângulos de ataque menores e grandes espessuras de corte.

6. Em geral, a força paralela média foi maior para os corpos de prova com orientação radial, que para os de orientação tangencial, confirmando a anisotropia transversal nas propriedades mecânicas.

7. O efeito da espessura de corte na força normal é dependente do ângulo de ataque utilizado.

8. Para o Eucalyptus grandis, a força normal média passou de positiva a negativa entre os ângulos de 20 e $30^{\circ}$, ou seja, passou pelo valor ótimo (zero) em um ângulo entre 20 e $30^{\circ}$, para os corpos de prova com orientação radial e tangencial.

9. Para o Eucalyptus saligna e Eucalyptus citriodora, a força normal média foi sempre negativa para todos os ângulos de ataque e espessuras de corte e para ambas as orientações dos corpos de prova.

\section{REFERÊNCIAS BIBLIOGRÁFICAS}

ASSOCIAÇÃO BRASILEIRA DE NORMAS TÉCNICAS. NBR7190/97 - Projeto de estruturas de madeira. Rio de Janeiro, ABNT, 1997.
FRANZ, N.C. An Analysis of the wood-cutting process. Michigan, EUA: University Michigan, Ann Arbor, 1958. 152p. Ph.D. Thesis

GONÇALVES, M.T.T. Proposta de metodologia para medição dos esforços de corte na usinagem da madeira. São Carlos: Escola de Engenharia de São Carlos - USP 1993. 249p. Tese Doutorado

HOADLEY, R.B. Understanding wood. A craftsman's guide to wood technology. The Taunton Press, p.256,1980.

KING, B.; FOSCHI, R.O. Crossed-ring dynamometer for direct force resolution into three orthogonal components. International Journal of Machine Tool Design Research. v.9, p. $345-356,1969$.

$\mathrm{KOCH}$, P. Utilization of hardwoods growing on southern pines sites. Volume II. Processing. USDA Forest Service, Agriculture Handbook, number 605 . Chapter 18, Machining, 1985, p.1687-2281.

McKENZIE, W.M;. Fundamental aspects of the wood cutting process. Forest Products Journal, v.10, n.9, p.447-456, 1960.

NÉRI, A.C.; GONÇALVES, R.; HERNANDEZ, R.E., Forças de corte ortogonal 90-0 em três espécies de madeira de eucalipto. Revista Brasileira de Engenharia Agrícola e Ambiental, Campina Grande, v.3, n.2, p. 239-244, 1999.

STEWART, H.A. Chip formation when orthogonally cutting wood against the grain. Wood Science, v.3 n.4, p. 193-203, 1971a

STEWART, H.A. Rake angle for planing hard maple determined best by depth of chipped grain. USDA Forest Service, Research Note NC-92, 1971b, 4p.

STEWART, H.A. Optimum rake angle related to selected properties of wood. Forest Products Journal. v.27, n.1, p.51-53, 1977.

WOODSON, G.E. Tool forces and chip types in orthogonal cutting of southern hardwoods. Research Paper SO-146, U.S. Department of Agriculture, Forest Service, 1979. 